\title{
Identification of miRNA-Target Gene Pairs Responsive to Fusarium Wilt of Cucumber via an Integrated Analysis of miRNA and Transcriptome Profiles
}

\author{
Jun Xu ${ }^{1}$, Qianqian Xian ${ }^{1}$, Ningyuan Zhang ${ }^{1}$, Ke Wang ${ }^{1}$, Xin Zhou ${ }^{1}$, Yansong Li ${ }^{1}$, Jingping Dong ${ }^{1}$ \\ and Xuehao Chen $1,2, *$ \\ 1 School of Horticulture and Plant Protection, Yangzhou University, Yangzhou 225009, China; \\ 006963@yzu.edu.cn (J.X.); QQxian1996@163.com (Q.X.); ningyuan1214@foxmail.com (N.Z.); \\ WK19825301461@163.com (K.W.); ZX957476929@163.com (X.Z.); liyansong202108@163.com (Y.L.); \\ jpdong@yzu.edu.cn (J.D.) \\ 2 State Key Laboratory of Vegetable Germplasm Innovation, Tianjin 300192, China \\ * Correspondence: xhchen@yzu.edu.cn
}

check for updates

Citation: Xu, J.; Xian, Q.; Zhang, N.; Wang, K.; Zhou, X.; Li, Y.; Dong, J.;

Chen, $X$. Identification of

miRNA-Target Gene Pairs

Responsive to Fusarium Wilt of

Cucumber via an Integrated Analysis of miRNA and Transcriptome Profiles. Biomolecules 2021, 11, 1620. https:// doi.org/10.3390/biom11111620

Academic Editors: Elena Khlestkina and Yuri Shavrukov

Received: 11 September 2021

Accepted: 28 October 2021

Published: 2 November 202

Publisher's Note: MDPI stays neutral with regard to jurisdictional claims in published maps and institutional affiliations.

Copyright: (c) 2021 by the authors. Licensee MDPI, Basel, Switzerland. This article is an open access article distributed under the terms and conditions of the Creative Commons Attribution (CC BY) license (https:/ / creativecommons.org/licenses/by/ $4.0 /)$.

\begin{abstract}
Fusarium wilt (FW) of cucumber (Cucumis sativus L.) caused by Fusarium oxysporum $\mathrm{f}$. sp. cucumerinum (Foc) is a destructive soil-borne disease that severely decreases cucumber yield and quality worldwide. MicroRNAs (miRNAs) are small non-coding RNAs (sRNAs) that are important for regulating host immunity because they affect target gene expression. However, the specific miRNAs and the miRNA/target gene crosstalk involved in cucumber resistance to $F W$ remain unknown. In this study, we compared sRNA-seq and RNA-seq data for cucumber cultivar 'Rijiecheng', which is resistant to $F W$. The integrated analysis identified $F W$-responsive miRNAs and their target genes. On the basis of verified expression levels, we detected two highly expressed miRNAs with down-regulated expression in response to Foc. Moreover, an analysis of 21 target genes in cucumber inoculated with Foc indicated that JRL3 (Csa2G362470), which is targeted by miR319a, and BEE1 (Csa1G024150), DAHP1 (Csa2G369040), and PERK2 (Csa4G642480), which are targeted by $m i R 6300$, are expressed at high levels, but their expression is further up-regulated after Foc inoculation. These results imply that miR319a-JRL3, miR6300-BEE1, miR6300-DAHP1 and miR6300$P E R K 2$ regulate cucumber defenses against $F W$, and provide the gene resources that may be useful for breeding programs focused on developing new cucumber varieties with enhanced resistance to $F W$.
\end{abstract}

Keywords: cucumber; miRNA; transcriptome; Fusarium wilt; resistance

\section{Introduction}

Cucumber (Cucumis sativus L.) is an important vegetable crop cultivated worldwide [1] Fusarium wilt $(F W)$ of cucumber, which is a typical and destructive soil-borne disease caused by Fusarium oxysporum f. sp. cucumerinum (Foc), is one of the major factors restricting global cucumber yield and quality [2-5].

During an infection, Foc easily penetrates cucumber plants and quickly spreads to the vascular tissues, where it occludes xylem vessels and produces a toxin that kills host cells, ultimately leading to $F W$. The disease results in the wilting of leaves or even the entire plant, with plant death occurring several days or weeks after infection [6,7]. Fusarium wilt is extremely difficult to control because it can occur during all cucumber growth stages [2]. Additionally, Foc can survive in the soil, straw or seeds for many years or even decades, leading to long-term disease cycles [8,9]. Furthermore, the changeable pathogenicity of Foc has limited the effectiveness of certain fungicides $[3,10]$. The most sustainable way to control FW involves the development and application of resistant cultivars. Thus, effective resistance genes need to be identified and the mechanism mediating disease resistance must be thoroughly characterized to develop FW-resistant cucumber cultivars. 
Plant microRNAs (miRNAs) are endogenous small non-coding RNAs (sRNAs) that serve as crucial molecular regulators of the expression of functional genes by targeting mRNAs or by inhibiting translation [11-13]. Plant miRNAs participate in almost all biological processes, such as growth and development [14,15], hormone signal transduction [16] and responses to abiotic and biotic stresses (e.g., pathogen attack) $[17,18]$. For example, in rice, overexpression of Osa-miR812w increases resistance to Magnaporthe oryzae, whereas CRISPR/Cas9-mediated miR812w editing enhances disease susceptibility, suggesting that miR812w positively contributes to blast resistance [18]. In cotton, cleavage of GhNAC100 mRNA by ghr-miR164 decreases the amount of GhNAC100 that can bind to the CGTA-box of GhPR3 promoter to repress expression, thereby enhancing the resistance to Verticillium dahliae [19]. Tomato $\ln c R N A 23468$ functions as a decoy RNA for miR482b to modulate the expression of NBS-LRR genes, resulting in increased resistance to Phytophthora infestans [20]. These results suggest that miRNAs mediate crucial plant defense gene regulatory pathways. Therefore, resistance-related miRNAs must be identified and their target genes associated with responses to pathogens should be functionally characterized. However, to date, very few miRNA-seq and RNA-seq analyses of cucumber defense responses to $F W$ have been conducted. Thus, the miRNAs associated with $F W$ resistance and their underlying mechanisms in cucumber remain unknown.

On the basis of an earlier examination of germplasm resources inoculated with Foc, we had confirmed that the cucumber cultivar 'Rijiecheng' was high-resistant against Foc, and might possess the resistant genes performing Foc defense [21]. In this study, we performed the integrated miRNA-seq and RNA-seq analyses to identify the $F W$-responsive miRNAs and their target genes in the $F W$-resistant cucumber cultivar 'Rijiecheng'. Through the miRNA-seq from the Rijiecheng roots infected with Foc, we identified that eleven differentially expressed miRNAs (DEMs) were related to $F W$ resistance. We further found that two miRNAs (miR319a and miR6300) had high expression levels and were downregulated expression affected by the inoculation with Foc. Followed the RNA-seq analysis of Rijiecheng infected with Foc, 21 corresponding target genes of miR319a and miR6300 had adverse expression tendencies and were expressed differently in cucumber roots inoculated with Foc. Based on the expression verification, we determined that JRL3 (Csa2G362470), which is putatively targeted by miR319a, and BEE1 (Csa1G024150), DAHP1 (Csa2G369040), and PERK2 (Csa4G642480), which are putatively targeted by miR6300, were abundantly expressed, with expression levels that were higher than those in the controls. These findings indicate that miR319a-JRL3, miR6300-BEE1, miR6300-DAHP1 and miR6300-PERK2 might be important for cucumber defenses against $F W$. Moreover, they may be useful for generating $F W$-resistant cucumber cultivars via breeding.

\section{Materials and Methods}

\subsection{Plant Materials and Foc Treatment}

This study was conducted using cucumber cultivar 'Rijiecheng', which was confirmed to be resistant to Foc during an earlier examination of germplasm resources inoculated with Foc [21]. Seeds were germinated on wet gauze in a Petri dish at $28^{\circ} \mathrm{C}$. The resulting seedlings were incubated in a growth chamber with the same conditions of $25^{\circ} \mathrm{C} / 18^{\circ} \mathrm{C}$ cycle under a $16 \mathrm{~h}$ light $/ 8 \mathrm{~h}$ dark cycle.

The Foc strain used in this study was isolated from cucumber roots exhibiting $F W$ symptoms and then propagated on potato dextrose agar with different antibiotics inhibiting bacteria growth in plates at $28{ }^{\circ} \mathrm{C}$ for 4 days. The conidia were harvested and isolated for several times. Followed the inoculation phenotypes and sequencing verification, the Foc strain was confirmed and then cultured in potato dextrose broth in plates on a shaker $(180 \mathrm{rpm})$ at $28{ }^{\circ} \mathrm{C}$ for 3 days. The concentration was adjusted to $10^{6}$ spores $/ \mathrm{mL}$ using sterile distilled water prior to the inoculation of 14-day large cucumber seedlings at second true-leaf stage via a published dip-inoculation method [22]. Three replicates of seedling roots were harvested at $0,24,48,96$, and $192 \mathrm{~h}$ after the Foc inoculation. All samples were flash-frozen in liquid nitrogen and stored at $-80{ }^{\circ} \mathrm{C}$ until analyzed. 


\subsection{Total RNA Isolation and Library Preparation for sRNA Sequencing}

We collected three replicates of Foc-inoculated cucumber roots at different postinoculation time-points $(0,48$, and $96 \mathrm{~h})$; non-inoculated roots were collected as the controls refer to the method as described by Dong and associates (2020) [22]. Total RNA for the real-time polymerase chain reaction (PCR) was extracted from flash-frozen cucumber roots using the MiniBEST Plant RNA Extraction Kit (TaKaRa, Dalian, China), after which RNA degradation and contamination were monitored on $2 \%$ agarose gels. Additionally, RNA purity was assessed using the NanoPhotometer ${ }^{\circledR}$ spectrophotometer (IMPLEN, Calabasas, CA, USA), whereas the RNA concentration was determined using the Qubit ${ }^{\circledR}$ RNA Assay Kit and the Qubit ${ }^{\circledR}$ 2.0 Fluorometer (Life Technologies, Gaithersburg, MD, USA).

For each sample, $3 \mu \mathrm{g}$ total RNA was used as the input material for constructing sRNA libraries. The sequencing libraries were generated using the NEBNext ${ }^{\circledR}$ Multiplex Small RNA Library Prep Set for Illumina ${ }^{\circledR}$ (New England Biolabs, Beverly, MA, USA) and index codes were added to attribute sequences to each sample. The sRNA sequencing analysis was completed using the AllPrep DNA-RNA-miRNA Universal kit (Qiagen, Duesseldorf, Germany), with DNA contaminants removed by an on-column DNase treatment.

\section{3. miRNA-Seq Analysis}

The miRNA sequencing analysis was performed by Novogene using the Illumina NextSeq 500 system. Expression levels were calculated as the number of transcripts per million (TPM). To identify the known miRNAs, the clean reads were used as queries for a BLAST search of the miRNA database miRbase 21.0 (http://www.mirbase.org/, accessed on 7 September 2021). The sRNA tags were mapped to the reference sequence using Bowtie (with no mismatches) to analyze their expression and distribution on the reference sequence [23]. We analyzed the miRNA families and their sequence conservation in miRbase, and the DEMs were further screened according to the following criterion: $\mid \log _{2}$ FPKM (fold-change) $\mid>1$ and $p$ value $<0.05$. For the unannotated sequences, we predicted new miRNAs using mireap (http:/ / sourceforge.net/projects/mireap/, accessed on 7 September 2021). As miRNAs are primarily bound to the target site by complementary pairing, the data were analyzed using Miranda (http:/ / www.microrna.org/microrna/ home.do, accessed on 7 September 2021) to identify the targets of the mature miRNA sequences. The sRNA raw data were deposited in the NCBI Sequence Read Archive (accession number PRJNA760453).

\subsection{MicroRNA Family Identification and Target Prediction}

The miRNA families in other species were identified. In our analysis pipeline, known miRNAs were used along with miRNA.dat (http:/ / www.mirbase.org/ftp.shtml, accessed on 7 September 2021) to search for families, whereas novel miRNA precursors were submitted to Rfam (http: / /rfam.xfam.org/, accessed on 7 September 2021) to screen for Rfam families. The miRNA expression levels were estimated as the number of TPM according to the following criteria [24]. The differential expression between two conditions/groups was analyzed using the DESeq R package (1.8.3). The $p$-values were adjusted according to the Benjamini and Hochberg method. A corrected $p$-value of 0.05 was set as the threshold for determining significant differences in expression. The target genes of miRNAs in plants were predicted using psRobot_tar in psRobot [25].

The TargetFinder software was used to predict miRNA target genes [26]. The candidate target genes of the DEMs were functionally characterized by a Gene Ontology (GO) enrichment analysis on the basis of the genome annotate of cucumber (http:/ / cucurbitgenomics. org/organism/20, accessed on 7 September 2021).

\subsection{Quantitative Real-Time RT-PCR ( $q$ RT-PCR) Assay of the miRNAs}

The known and novel miRNAs were assayed by qPCR to validate relative expression patterns. The reverse transcription reaction was performed using the miRNA 1st Strand cDNA Synthesis Kit (by stem-loop) (Vazyme, Nanjing, China). The qPCR analysis was 
performed using the miRNA Universal SYBR ${ }^{\circledR}$ qPCR Master Mix (Vazyme, Nanjing, China), with U6 snRNA used as the internal control. The miRNAs gene expression was calculated using qRT-PCR analysis, and expression data represent as the $2^{-\Delta \mathrm{Ct}}$ method followed by further statistical analysis [27]. The standard deviation was measured for three biological replicates. The primers of the miRNAs used in the experiment were designed by miRNA Design V1.01 software and listed in Additional file 1: Table S1.

\subsection{Identification and Validation of Target Differentially Expressed Target Genes}

We had analyzed the transcriptome of Foc-inoculated cucumber roots collected at different time-points after inoculation (0, 24, 48, 96, and $192 \mathrm{~h})$. The generated data were submitted to the NCBI database (accession number PRJNA472169). The differentially expressed genes were considered with an adjusted FDR $<0.01$ identified by DESeq and $\mid \log _{2}$ FPKM (fold-change) $\mid \geq 1$. The transcriptome was used to further screen for target genes of the miRNAs. Differences in the expression of the candidate genes were indicated by the color scale of the Toolbox for Biologists software.

The expression verification of the candidate genes was performed by the qRT-PCR analysis in the cucumber cultivar 'Rijiecheng' infected with Foc. The RNA samples of the Foc-inoculated cucumber roots were reverse transcribed into cDNA using the HiScript Q RT SuperMix for qPCR (Vazyme, Nanjing, China). The target gene-specific qRT-PCR primers were designed using the Beacon Designer 7.0 software. The cucumber tubulin alpha chain gene (Csa4G000580) was used as the internal reference control. The qRT-PCR analysis was performed using the Iqtm5 Multicolor qPCR detection system (Bio-Rad, Hercules, CA, USA) and the AceQ SYBR Green Master Mix (Vazyme, Nanjing, China), with three technical replicates per biological replicate. The expression data for three biological replicates were analyzed and are presented herein as the mean \pm standard deviation. Primer information is provided in Additional file 1: Table S1.

\section{Results}

\subsection{Identification of miRNAs Responsive to Foc}

To identify the miRNAs involved in plant responses to Foc, 15 sRNA libraries for three biological replicates of the Foc-inoculated roots at 48 and $96 \mathrm{~h}$ post-inoculation and the non-inoculated controls were prepared for a high-throughput sequencing analysis to identify miRNAs. We used the 18- to 24-nt sRNA sequences as queries to search for matches among the plant miRNA sequences in the miRBase 21.0 database. A total of 1185 miRNAs, including 1115 known miRNAs and 70 novel miRNAs, were identified in all the samples (Figure 1A). On the basis of detecting significant differences in expression in different groups using various methods, we further identified six differentially expressed miRNAs (DEMs) between the inoculated roots (R_48h_F) and the control (mock-inoculated) roots (R_48h_C) at $48 \mathrm{~h}$ post-inoculation, and seven DEMs between the inoculated roots (R_96h_F) and the control roots (R_96h_C) at $96 \mathrm{~h}$ post-inoculation, including two overlap DEMs in the two compare combinations (Figure 1B). The expression of these 11 independent miRNAs was significantly induced by Foc with the expression value from the different sequencing libraries visualized according to the changing colors (Figure $1 \mathrm{C}$ ), suggesting these DEMs may be important for cucumber defense responses to $F W$.

\subsection{Validation of miRNA Expression by $q R T-P C R$}

To validate the identified DEMs, we completed a qRT-PCR assay to analyze the miRNA expression levels in the Foc-inoculated and control cucumber roots at three post-inoculation time-points (0, 48 and $96 \mathrm{~h})$. The expression trends of the 11 selected miRNAs revealed by qRT-PCR were consistent with the sRNA sequencing data, and further reflected the reliability of the sRNA sequencing data obtained in this study (Figure 2). Among them, the five miRNAs contained the miR6300, miR398, miR398-3p miR319a and miR319a-3p had higher expression levels relative to other miRNAs and were obviously induced after inoculation with Foc. We aimed to use the $F W$-resistant cultivar 'Rijiecheng' to identify the 
resistant genes, and the accumulating evidence showed that miRNAs negatively regulate the corresponding target genes to defend against the disease infection [20]. Hence, we selected the miR319a and miR6300 that had high expression value and were significantly down-regulated expression relative to the mock-inoculated controls, suggesting that they might regulate the increased expression of defense-related genes to enhance the resistance of cucumber cultivar 'Rijiecheng' to $F W$.

A
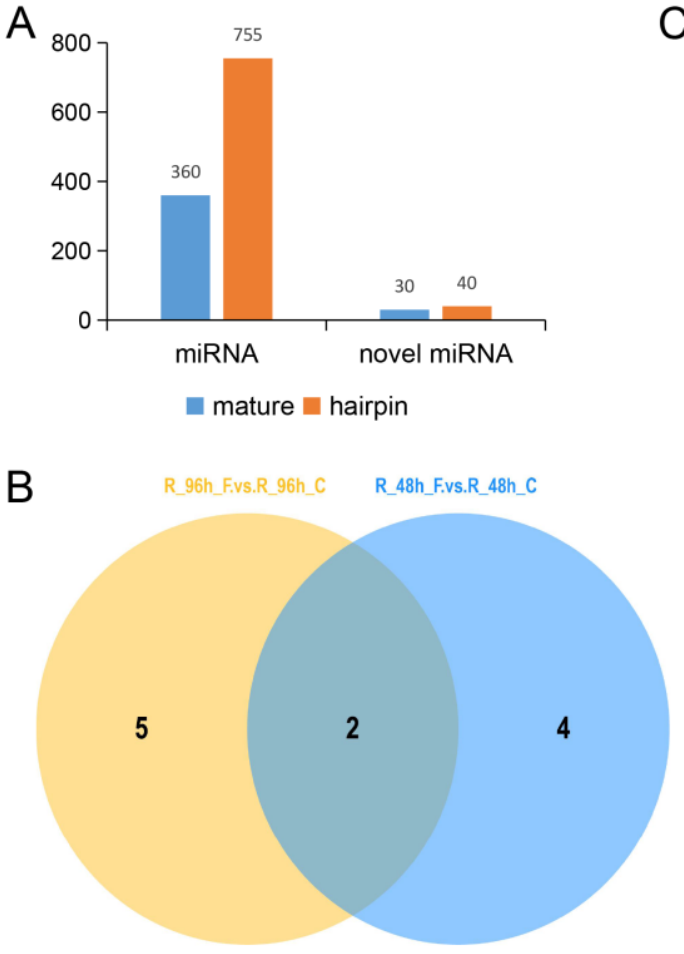

C

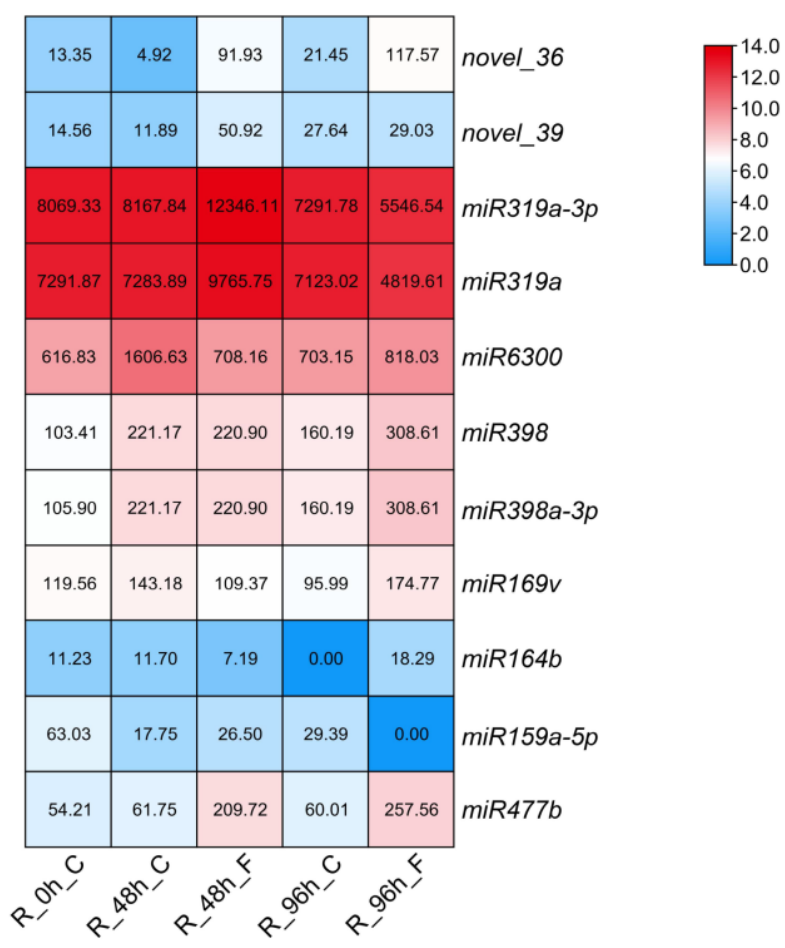

Figure 1. Identification of differentially expressed miRNAs (DEMs) responsive to $F W$ in cucumber. (A) Distribution of known and novel cucumber miRNAs, including mature and hairpin miRNAs; (B) Venn diagram of the DEMs between the Foc-inoculated cucumber roots and the mock-inoculated control roots at 48 and $96 \mathrm{~h}$ post-inoculation; (C) Heatmap analysis of DEMs among different sequencing libraries. Red and blue indicate up-regulated and down-regulated expression, respectively.

\subsection{Identification of miRNA Target Genes and Profile Analyses}

To further clarify the miRNA-mediated regulatory networks in cucumber inoculated with Foc, we predicted the target genes of miRNAs using psRobot_tar in psRobot [25]. The 1147 target genes identified for the 11 DEMs were subsequently analyzed on the basis of the transcriptome data for the 'Rijiecheng' samples inoculated with Foc. The miR319a and miR6300 respectively possessed 51 and 501 target genes, and among them, 273 genes were further performed classification based on the functional analysis of the GO enrichment with the $p$ value $<0.05$ and were mainly classified in 14 groups (e.g., protein binding, protein kinase activity, transcription factors, oxidoreductase activity) associated with diversity functions (http:/ / cucurbitgenomics.org/goenrich, accessed on 7 September 2021). However, the function characteristics of other genes were not annotated (Figure 3, Additional file 2: Table S2). Based on the screening of differentially expressed genes via transcriptome of cucumber cultivar 'Rijiecheng' infected with Foc, we identified that 21 target genes were obviously affected by Foc. We also analyzed the association between miR6300 and miR319a and their target genes via a functional characterization based on the bioinformatics method (Figure 4A, Additional file 3: Table S3). Moreover, the expression of these 21 target genes was significantly up-regulated induced by Foc with the expression data from the different transcriptome libraries visualized according to the changing colors, in contrast to the miRNA expression trends (Figure 4B), indicative of a positive role for these up-regulated genes in cucumber cultivar 'Rijiecheng' defense responses to $F W$. 

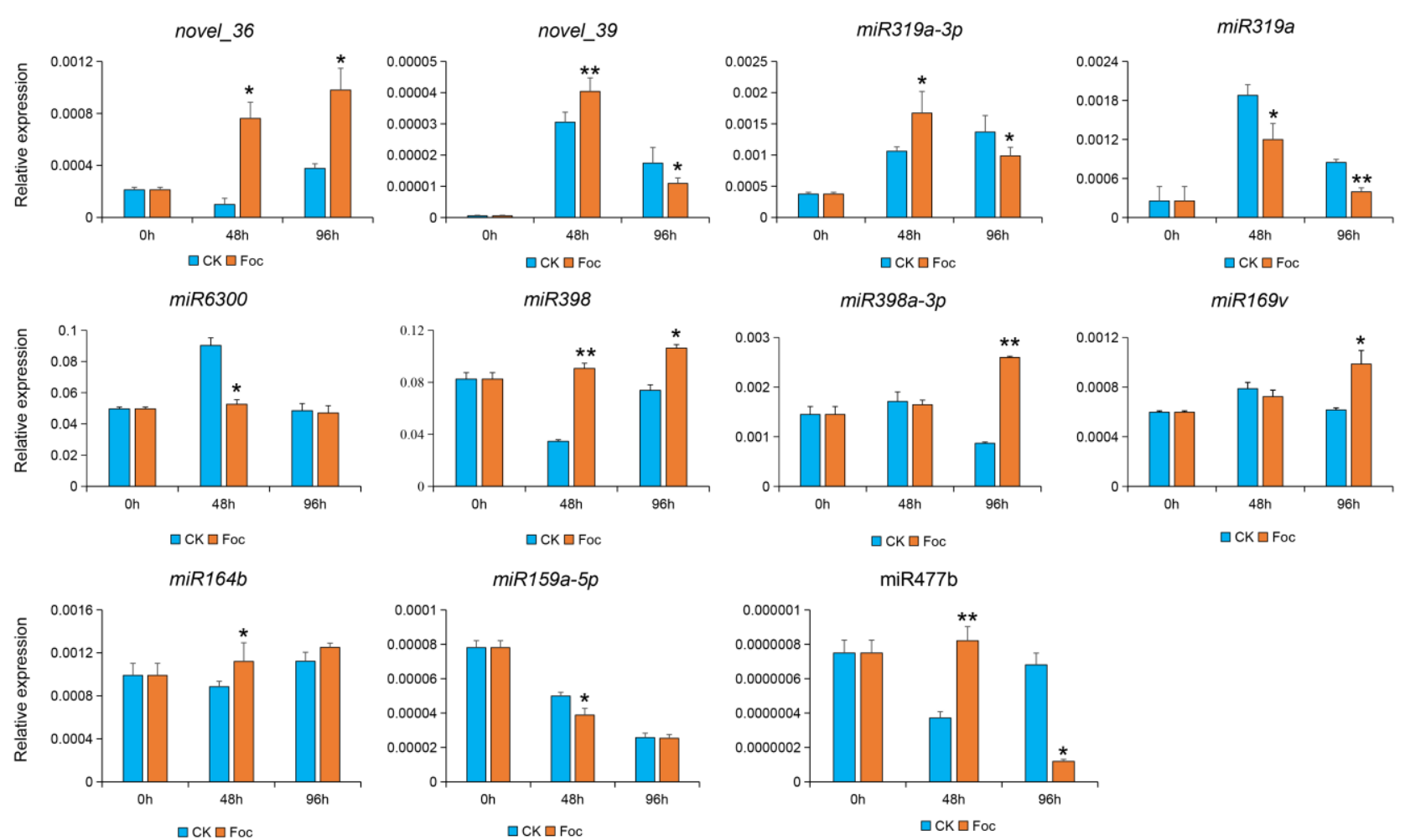

Figure 2. Validation of 11 DEMs in cucumber roots infected with Foc by stem-loop qPCR. Relative miRNA expression levels in the Foc-inoculated cucumber roots and the mock-inoculated control roots at 48 and $96 \mathrm{~h}$ post-inoculation were calculated according to the $2^{-\Delta \mathrm{Ct}}$ method, with U6 snRNA used as the internal reference control. Data are presented as the mean \pm standard deviation of three biological replicates. *: significantly different at $p<0.05 ;{ }^{* *}$ : significantly different at $p<0.01$.

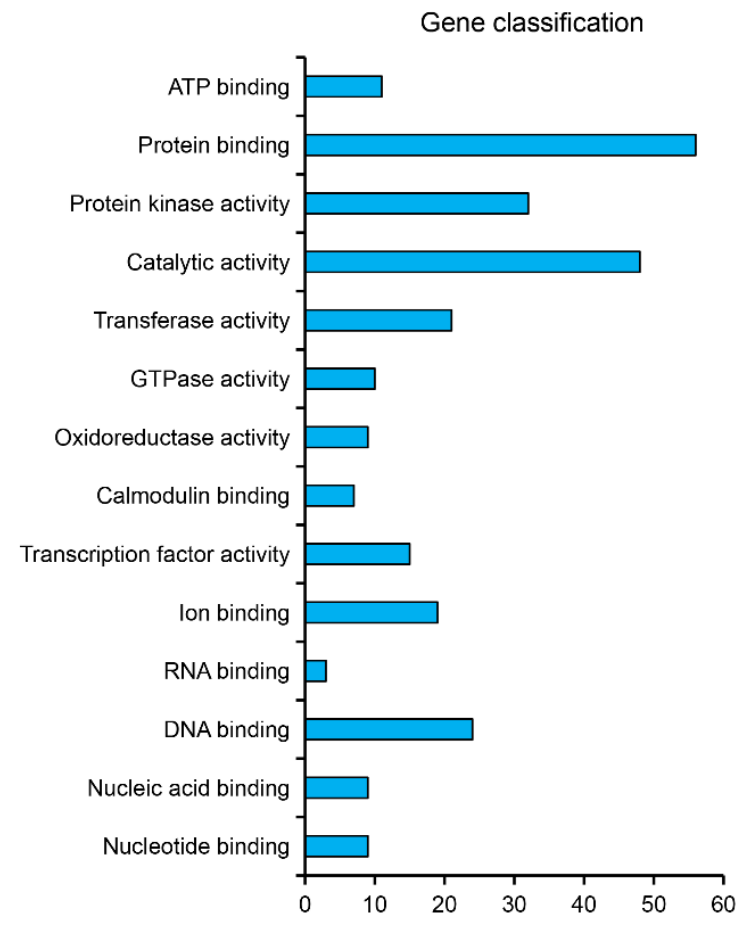

Figure 3. Functional classification of the targets of the miR319a and miR6300. The gene classification analysis was on the basis of GO enrichment. The ordinate represents the gene functions, and abscissa represents the number of genes. 
A

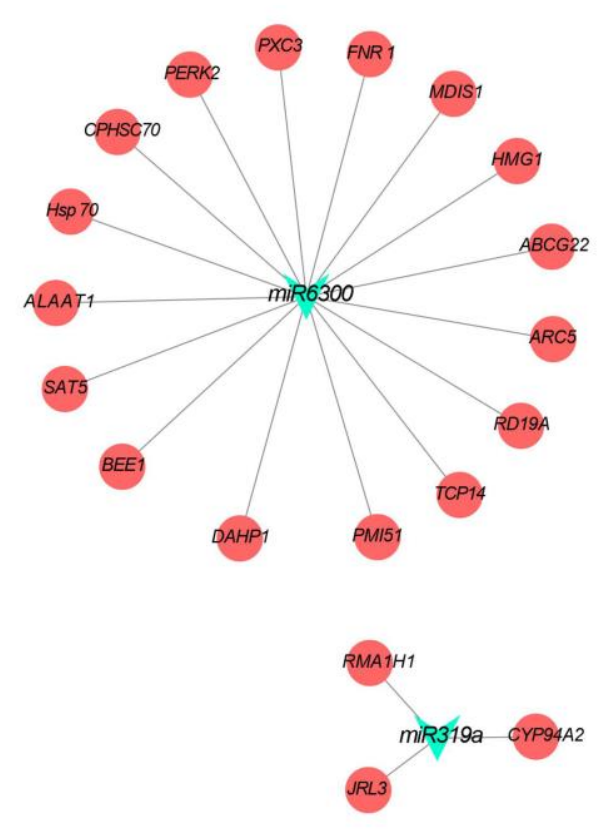

B

\begin{tabular}{|c|c|c|c|c|c|c|}
\hline 80.30 & 121.25 & 116.76 & 80.42 & 38.95 & $J R L 3$ & 10.0 \\
\hline 11.44 & 16.05 & 27.13 & 16.39 & 17.90 & $R M A 1 H 1$ & 8.0 \\
\hline 4.22 & 3.59 & 9.26 & 40.57 & 7.75 & CYP94A2 & 0 \\
\hline 7.40 & 12.35 & 15.88 & 15.74 & 8.21 & TCP14 & 1.0 \\
\hline 12.59 & 9.73 & 54.05 & 110.04 & 6.99 & BEE1 & \\
\hline 15.79 & 16.08 & 32.84 & 30.72 & 22.03 & SAT5 & \\
\hline
\end{tabular}

Figure 4. Target genes of selected miRNAs analyzed on the basis of the transcriptome of cucumber inoculated with Foc. (A) Predicted regulatory networks between miR6300 and miR319a and the differentially expressed target genes; (B) Analysis of target gene expression profiles in cucumber roots after the Foc inoculation. 'Rijiecheng' roots inoculated with Foc collected at different post-inoculation time-points $(0,24,48,96$ and $192 \mathrm{~h})$ were used to analyze the target gene expression patterns. Genes more highly or more weakly expressed in the roots were colored red and blue, respectively. The RNA-seq data were submitted to the NCBI database (accession number PRJNA472169).

\subsection{Verification of the Expression of Target Genes in Cucumber Infected with Foc}

To confirm the Foc-induced expression of candidate target genes, the expression patterns revealed by the transcriptome analysis of cucumber roots infected with Foc were investigated by qRT-PCR analysis. We found that the expression of 21 target genes of two miRNAs were up-regulated after the Foc inoculation relative to the mock-inoculated controls, and these genes were highly or weakly expressed in the roots colored red and blue, respectively (Figure 5A). In detail, among them, the JRL3 gene with a putative target site of miR319a as well as the BEE1,DAHP1, and PERK2 genes with a putative target site of miR6300 were better detected by a miRNA-target gene matching analysis. Additionally, these four genes had higher expression levels relative to the others and were significantly up-regulated affected by Foc at certain post-inoculation time-points, relative to the control levels (Figure $5 \mathrm{~B}$ ), indicating that these miRNA-target pairs might participate in cucumber defenses against $F W$. 
A

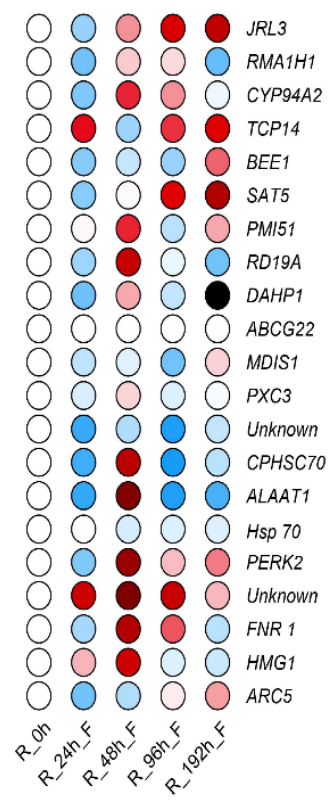

B
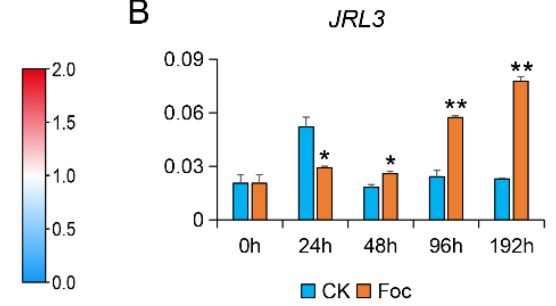

miR319a 5' UUGG -ACUGAAGGGAGCUCCCU 3' JRL3 3'AACCAUGAUUUCCCCC AAGGAA 5'

DAHP1

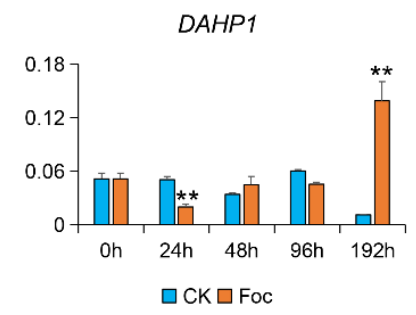

miR6300 5' GUCGUUGUAGUAUAGUGG 3'

DAHP1 3' CAGGAACAUUAUAAUAGG 5'

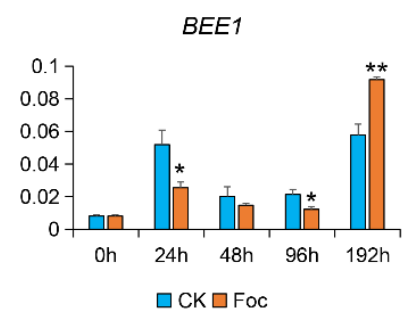

miR6300 5' GGUGAUAUGAUGUUGCUG 3' BEE1 3' CCAAUAuacuauaAcuau 5'

PERK2

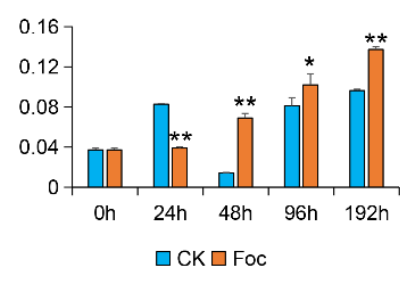

miR6300 5' GGUGAUAUG -AUGUUGCUG 3' PERK2 3'CAACUAUAAACACGACGAC 5'

Figure 5. Validation of the target genes in cucumber inoculated with Foc. (A) Target gene expression levels in the Focinoculated cucumber roots and the mock-inoculated control roots were calculated according to the $2^{-\Delta \Delta \mathrm{Ct}}$ method. Genes more highly or more weakly expressed in the roots were colored red and blue, respectively; (B) Expression patterns of four candidate target genes as well as the matches between miR6300 and miR319a and selected target genes. Data are presented as the mean \pm standard error of three biological replicates. *: significantly different at $p<0.05 ; * *$ : significantly different at $p<0.01$.

\section{Discussion}

Fusarium wilt is a serious factor restricting global cucumber productivity $[2,3]$. The most sustainable method to control FW disease is the use of resistant cultivars to mine disease-resistance genes and better investigate disease-resistance mechanisms to develop FW-resistant cucumber cultivars.

Increasing research attention focus on the utility of functional miRNAs that directly regulate target genes for modulating host immune responses to enhance fungal disease resistance. For example, cotton $g h r-m i R 164$ cleaves GhNAC100 mRNA to regulate the expression of downstream disease-related genes and increase plant resistance to Verticillium wilt [19]. Additionally, in cotton, miR5272a-GhMKK6, miR414c-GhFSD1 and miR477CBP60A help regulate plant defenses against pathogens [28-30]. In potato, over-expression of miR482e results in the silencing of NBS-LRR protein-encoding genes and enhanced plant sensitivity to $V$. dahliae infections [31]. Other studies proved that $m i R 482 b$ negatively regulates tomato resistance to $P$. infestans [20,32]. In the model plant Arabidopsis thaliana, many miRNA regulatory networks, including those involving miR156-SPL9, miR396-GRF, miR400-PPR, miR472-RDR6, miR773-MET2, miR844-CDS3 and miR858-MYB, mediate the resistance to pathogens [33-39]. In rice, miRNAs with functions influencing blast resistance have been reported, including miRNAs that positively (miR7695, miR160, miR398, miR162, and miR166k-166h) and negatively (miR156, miR164, miR167, miR168, miR169, miR319, miR396 and miR1873) regulate immune responses [17,40-51]. Many miRNAs or target genes have been applied to protect various transgenic plants against diseases. However, to date, there have been relatively few reports describing miRNAs or their regulatory networks associated with cucumber immunity to $F W$.

Based on an earlier examination of germplasm resources inoculated with Foc, we had determined that the cucumber cultivar 'Rijiecheng' was highly resistant against Foc and might possess the resistant genes performing Foc defense [21]. Hence, we performed a comparative analysis of miRNA and transcript profiles in cucumber cultivar 'Rijiecheng' 
roots inoculated with Foc, we wanted to identify the DEMs and their target genes related to the $F W$ responsive to better investigate the resistant mechanism of the cultivar 'Rijiecheng'. In detail, we identified 11 independent DEMs from the miRNA sequencing (Figure 1), we further analyzed the expression patterns of these miRNAs in the Rijiecheng roots infected with Foc (Figure 2). Accumulating evidence shows that miRNAs negatively regulate the corresponding target genes to defend against the disease infection [20,31,32]. Therefore, we selected the miR319a and miR6300 that had high expression levels and were obviously down-regulated after Foc infection, suggesting that these two miRNAs might affect the up-regulated expression of their target genes in the cucumber cultivar Rijiecheng to defense FW disease. The target genes of miR319a and miR6300 were further screened by the transcriptome of Rijiecheng roots infected with Foc, and found that 21 genes differentially expressed after Foc attack (Figure 4). We further verified the disease-related variations in miRNAs and target genes, and revealed that the expression levels of miR319a and miR6300 and their target genes JRL3, BEE1, DAHP1 and PERK2 are obviously affected by Foc (Figures 2 and 5). Furthermore, the regulatory networks or functions of these two modules will be further investigated with phenotypes by the cucumber transgenic method. The other up-regulated miRNAs and their target genes will be further verified in the FW-susceptible cultivars and better identify the susceptibility genes to analyze the molecular mechanism of susceptibility of cucumber against $F W$. In the future, the availability of efficient multiplex transgene-free systems using new genome editing tools will be performed to introduce the broad-spectrum $F W$ resistance by targeting multiple susceptibility genes simultaneously.

The miR319a-JRL3, miR6300-BEE1, miR6300-DAHP1 and miR6300-PERK2 pairs were confirmed that were obviously induced by the Foc. Additionally, the accumulated evidence shows that JRL3 contains three jacalin-like lectin domains, and additionally, the effects of JRL3 expression on plant defenses against pathogens have been investigated in several species. For example, in wheat (Triticum aestivum), TaJRLL1 encodes mannosespecific jacalin-like lectin domains and regulates the salicylic acid-dependent and jasmonic acid-dependent pathways during defense responses to the fungal pathogen Fusarium graminearum and the biotrophic fungal pathogen Blumeria graminis [52]. The BEE1 gene (brassinosteroid enhanced expression 1) encodes a bHLH domain. In soybean (Glycine max), the expression of a bHLH transcription factor gene, GmPIB1, is significantly induced by Phytophthora sojae, which results in the repressed expression of GmSPOD1 and enhanced reactive oxygen species production to increase plant resistance to $P$. sojae [53]. DAHP1 encodes a 3-deoxy-D-arabino-heptulosonate 7-phosphate synthase and significantly induce by pathogenic Pseudomonas syringae strains [54]. Considered together, these findings indicate that miR319a and miR6300 might affect JRL3, BEE1, DAHP1 and PERK2 expression accordingly to regulate the resistance of cucumber to $F W$. This information may be relevant for future studies conducted to elucidate the genetic basis of $F W$ resistance, with implications for the breeding of FW-resistant cucumber cultivar.

\section{Conclusions}

Based on a comparative analysis of sRNA-seq and RNA-seq data from the FW-resistant cucumber cultivar 'Rijiecheng' inoculated with Foc, we identified the Foc-responsive miRNAs and their target genes. Among them, miR319a and miR6300 were highly downregulated expression by the inoculation with Foc, and on the basis of RNA-seq analysis, 21 corresponding target genes were screened in cucumber roots inoculated with Foc. We further determined that JRL3, which is putatively targeted by miR319a, and BEE1, DAHP1 and PERK2, which are putatively targeted by $m i R 6300$, were abundantly expressed, with expression levels that were higher than those in the controls. These results revealed that miR319a-JRL3, miR6300-BEE1, miR6300-DAHP1 and miR6300-PERK2 pairs might be important for cucumber defenses against $F W$. 
Supplementary Materials: The following are available online at https: / www.mdpi.com/article/ 10.3390/biom11111620/s1, Additional file 1: Table S1. Information regarding the PCR primers, Additional file 2: Table S2. Functional classification of the targets of miR6300 and miR319a. Additional file 3: Table S3. Functional characterization of miR6300 and miR319a and differentially expressed target genes.

Author Contributions: Experiments were designed by X.C. Experiments were performed by J.X., Q.X., N.Z., K.W., X.Z., Y.L. and J.D.; J.X. and X.C. drafted and revised the manuscript. All authors have read and agreed to the published version of the manuscript.

Funding: This study was supported by the National Natural Science Foundation of China (31902015), Jiangsu Agricultural Science and Technology Innovation Fund (CX (19)3029), Natural Science Foundation of Jiangsu Province (BK20190887), and Jiangsu Agricultural Science and Technology Innovation Fund (CX (20)3165).

Institutional Review Board Statement: Not applicable.

Informed Consent Statement: Not applicable.

Data Availability Statement: The miRNA-Seq data associated with this study have been submitted to the NCBI SRA database (accession number PRJNA760453). Ethics approval and consent to participate, not applicable.

Conflicts of Interest: All the authors declare that they have no competing interest.

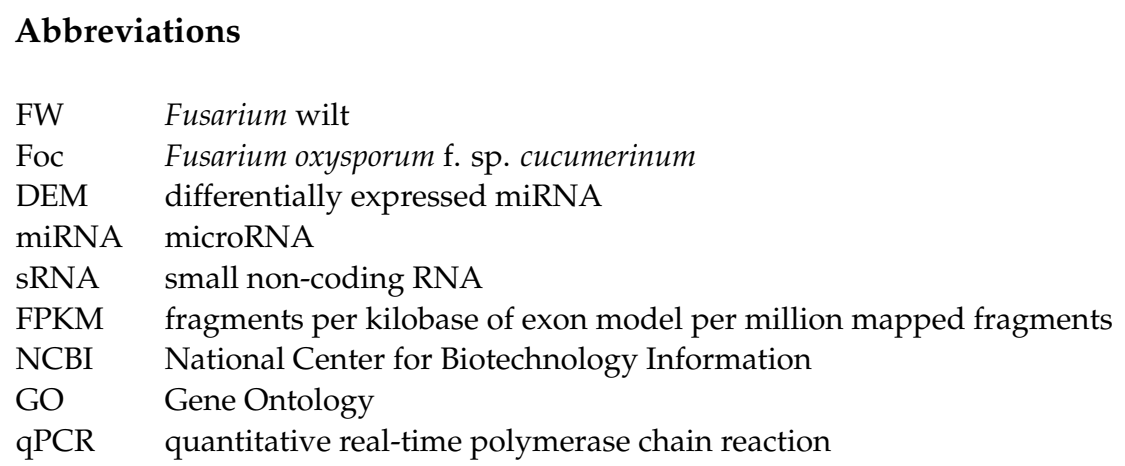

\section{References}

1. Huang, S.W.; Li, R.Q.; Zhang, Z.H.; Li, L.; Gu, X.; Fan, W.; Lucas, W.J.; Wang, X.; Xie, B.; Ni, P.; et al. The genome of the cucumber Cucumis sativus L. Nat. Genet. 2009, 41, 1275-1281. [CrossRef] [PubMed]

2. Ahn, P.; Chung, H.S.; Lee, Y.H. Vegetative compatibility groups and pathogenicity among isolates of Fusarium oxysporum f. sp. cucumerinum. Plant Dis. 1998, 82, 244-246. [CrossRef]

3. Huang, X.; Shi, D.; Sun, F.; Lu, H.; Liu, J.; Wu, W. Efficacy of sludge and manure compost amendments against Fusarium wilt of cucumber. Environ. Sci. Pollut. Res. Int. 2012, 19, 3895-3905. [CrossRef] [PubMed]

4. Zhang, S.P.; Miao, H.; Yang, Y.H.; Xie, B.Y.; Wang, Y.; Gu, X.F. A major quantitative trait locus conferring resistance to Fusarium wilt was detected in cucumber by using recombinant inbred lines. Mol. Breed. 2014, 34, 1805-1815. [CrossRef]

5. Wang, M.; Sun, Y.M.; Sun, G.M.; Liu, X.; Zhai, L.; Shen, Q.; Guo, S. Water balance altered in cucumber plants infected with Fusarium oxysporum f. sp. cucumerinum. Sci. Rep. 2015, 5, 7722. [CrossRef]

6. Li, P.; Pu, X.; Feng, B.; Yang, Q.; Shen, H.; Zhang, J.; Lin, B. FocVel1 influences asexual production, filamentous growth, biofilm formation, and virulence in Fusarium oxysporum f. sp. cucumerinum. Front. Plant Sci. 2015, 6, 312. [CrossRef] [PubMed]

7. McGovern, R.J. Management of tomato diseases caused by Fusarium oxysporum. Crop Prot. 2015, 73, 78-92. [CrossRef]

8. Snyder, W.C.; Hansen, H.N. The species concept in Fusarium. Am. J. Bot. 1940, 27, 64-67. [CrossRef]

9. Martínez, R.; Aguilar, M.I.; Guirado, M.L.; Alvarez, A.; Gomez, J. First report of Fusarium wilt of cucumber caused by Fusarium oxysporum in Spain. Plant Pathol. 2003, 52, 410. [CrossRef]

10. Chen, L.H.; Huang, X.Q.; Zhang, F.G.; Zhao, D.K.; Yang, X.M.; Shen, Q.R. Application of Trichoderma harzianum SQR-T037 bioorganic fertiliser significantly controls Fusarium wilt and affects the microbial communities of continuously cropped soil of cucumber. J. Sci. Food Agric. 2012, 92, 2465-2470. [CrossRef] [PubMed]

11. Tang, G.; Reinhart, B.J.; Bartel, D.P. A biochemical framework for RNA silencing in plants. Gene 2003, 17, 49-63. [CrossRef] [PubMed]

12. Chen, X.A. MicroRNA as translational repressor of APETALA2 in Arabidopsis flower development. Science 2004, 303, $2022-2025$. [CrossRef] [PubMed] 
13. Jones-Rhoades, M.W.; Bartel, D.P.; Bartel, B. MicroRNAs and their regulatory roles in plants. Annu. Rev. Plant Biol. $2006,57,19-53$. [CrossRef]

14. Sun, C.; Zhao, Q.; Liu, D.D. Ectopic expression of the apple Md-miRNA156h gene regulates flower and fruit development in Arabidopsis. Plant Cell Tissue 2013, 112, 343-351. [CrossRef]

15. Tang, F.; Wei, H.R.; Zhao, S.T. Identification of microRNAs involved in regeneration of the secondary vascular system in Populus tomentosa Carr. Front. Plant Sci. 2016, 7, 724-741. [CrossRef] [PubMed]

16. Chen, Z.H.; Bao, M.L.; Sun, Y.Z.; Yang, Y.J.; Xu, X.H.; Wang, J.H.; Han, N.; Bian, H.W.; Zhu, M.Y. Regulation of auxin response by miR393-targeted transport inhibitor response protein 1 is involved in normal development in Arabidopsis. Plant Mol. Biol. 2011, 77, 619-629. [CrossRef] [PubMed]

17. Sánchez-Sanuy, F.; Peris-Peris, C.; Tomiyama, S.; Okada, K.; Hsing, Y.I.; San Segundo, B.; Campo, S. Osa-miR7695 enhances transcriptional priming in defense responses against the rice blast fungus. BMC Plant Biol. 2019, 19, 563. [CrossRef]

18. Campo, S.; Sánchez-Sanuy, F.; Camargo-Ramírez, R.; Gómez-Ariza, J.; Baldrich, P.; Campos-Soriano, L.; Soto-Suárez, M.; San Segundo, B. A novel transposable element-derived microRNA participates in plant immunity to rice blast disease. Plant Biotechnol. J. 2021, 19, 1798-1811. [CrossRef] [PubMed]

19. Hu, G.; Lei, Y.; Liu, J.; Hao, M.; Zhang, Z.; Tang, Y.; Chen, A.; Wu, J. The ghr-miR164 and GhNAC100 modulate cotton plant resistance against Verticillium dahlia. Plant Sci. 2020, 293, 110438. [CrossRef]

20. Jiang, N.; Cui, J.; Shi, Y.; Yang, G.; Zhou, X.; Hou, X.; Meng, J.; Luan, Y. Tomato lncRNA23468 functions as a competing endogenous RNA to modulate NBS-LRR genes by decoying miR482b in the tomato-Phytophthora infestans interaction. Hortic. Res. 2019, 6, 28. [CrossRef] [PubMed]

21. Dong, J.P.; Qi, X.H.; Xu, Q.; Chen, X.H. Segregation and identification of Fusarium oxysporum f. sp. cucumerinum and analysis of cucumber varieties' resistance difference. Mol. Plant Breed. 2017, 15, 3648-3653.

22. Dong, J.P.; Wang, Y.A.; Xian, Q.Q.; Chen, X.; Xu, J. Transcriptome analysis reveals ethylene-mediated defense responses to Fusarium oxysporum f. sp. cucumerinum infection in Cucumis satious L. BMC Plant Biol. 2020, 20, 334. [CrossRef] [PubMed]

23. Langmead, B.; Trapnell, C.; Pop, M.; Salzberg, S.L. Ultrafast and memory-efficient alignment of short DNA sequences to the human genome. Genome Biol. 2009, 10, R25. [CrossRef]

24. Zhou, M.; Gu, L.; Li, P.; Song, X.; Cao, X. Degradome sequencing reveals endogenous small RNA targets in rice (Oryza sativa L. ssp. indica). Front. Biol. 2010, 5, 67-90. [CrossRef]

25. Wu, H.J.; Ma, Y.K.; Chen, T.; Wang, M.; Wang, X.J. PsRobot: A web-based plant small RNA meta-analysis toolbox. Nucleic Acids Res. 2012, 40, W22-W28. [CrossRef] [PubMed]

26. Fahlgren, N.; Howell, M.D.; Kasschau, K.D.; Chapman, E.J.; Sullivan, C.M.; Cumbie, J.S.; Givan, S.A.; Law, T.F.; Grant, S.R.; Dangl, J.L.; et al. High-throughput sequencing of Arabidopsis microRNAs: Evidence for frequent birth and death of MIRNA genes. PLoS ONE 2007, 2, e219. [CrossRef]

27. Gheysarzadeh, A.; Sadeghifard, N.; Afraidooni, L.; Pooyan, F.; Mofid, M.R.; Valadbeigi, H.; Bakhtiari, H.; Keikhavani, S. Serumbased microRNA biomarkers for major depression: MiR-16, miR-135a, and miR-1202. J. Res. Med. Sci. 2018, 23, 69. [CrossRef] [PubMed]

28. Wang, C.; He, X.; Wang, X.; Zhang, S.; Guo, X. ghr-miR5272a-mediated regulation of GhMKK6 gene transcription contributes to the immune response in cotton. J. Exp. Bot. 2017, 68, 5895-5906. [CrossRef] [PubMed]

29. Wang, W.; Liu, D.; Chen, D.; Cheng, Y.; Zhang, X.; Song, L.; Hu, M.; Dong, J.; Shen, F. MicroRNA414c affects salt tolerance of cotton by regulating reactive oxygen species metabolism under salinity stress. RNA Biol. 2019, 16, 362-375. [CrossRef] [PubMed]

30. Hu, G.; Hao, M.; Wang, L.; Liu, J.; Zhang, Z.; Tang, Y.; Peng, Q.; Yang, Z.; Wu, J. The cotton miR477-CBP60A module participates in plant defence against Verticillium dahliae. Mol. Plant Microbe Interact. 2019, 33, 624-636. [CrossRef] [PubMed]

31. Yang, L.; Mu, X.Y.; Liu, C.; Cai, J.; Shi, K.; Zhu, W.; Yang, Q. Over expression of potato miR482e enhanced plant sensitivity to Verticillium dahliae infection. J. Integr. Plant Biol. 2015, 57, 1078-1088. [CrossRef] [PubMed]

32. Soto-Suárez, M.; Baldrich, P.; Weigel, D.; Rubio-Somoza, I.; San Segundo, B. The Arabidopsis miR396 mediates pathogen-associated molecular pattern-triggered immune responses against fungal pathogens. Sci. Rep. 2017, 7, 44898. [CrossRef]

33. Boccara, M.; Sarazin, A.; Thiebeauld, O.; Jay, F.; Voinnet, O.; Navarro, L.; Colot, V. The Arabidopsis miR472-RDR6 silencing pathway modulates PAMP- and effector-triggered immunity through the post-transcriptional control of disease resistance genes. PLoS Pathog. 2014, 10, e1003883. [CrossRef] [PubMed]

34. Park, Y.J.; Lee, H.J.; Kwak, K.J.; Lee, K.; Hong, S.W.; Kang, H. MicroRNA400-guided cleavage of Pentatricopeptide repeat protein mRNAs Renders Arabidopsis thaliana more susceptible to pathogenic bacteria and fungi. Plant Cell Physiol. 2014, 55, 1660-1668. [CrossRef]

35. Lee, H.J.; Park, Y.J.; Kwak, K.J.; Kim, D.; Park, J.H.; Lim, J.Y.; Shin, C.; Yang, K.Y.; Kang, H. MicroRNA844-Guided Down-regulation of Cytidinephosphate Diacylglycerol Synthase3 (CDS3) mRNA Affects the Response of Arabidopsis thaliana to Bacteria and Fungi. Mol. Plant Microbe Interact. 2015, 28, 892-900. [CrossRef] [PubMed]

36. Camargo-Ramírez, R.; Val-Torregrosa, B.; San Segundo, B. MiR858-Mediated Regulation of Flavonoid-Specific MYB Transcription Factor Genes Controls Resistance to Pathogen Infection in Arabidopsis. Plant Cell Physiol. 2018, 59, 190-204. [CrossRef]

37. Salvador-Guirao, R.; Baldrich, P.; Weigel, D.; Rubio-Somoza, I.; San Segundo, B. The MicroRNA miR773 is involved in the Arabidopsis immune response to fungal pathogens. Mol. Plant Microbe Interact. 2018, 31, 249-259. [CrossRef] [PubMed] 
38. Chandran, V.; Wang, H.; Gao, F.; Cao, X.L.; Chen, Y.P.; Li, G.B.; Zhu, Y.; Yang, X.M.; Zhang, L.L.; Zhao, Z.X.; et al. miR396-OsGRFs module balances growth and rice blast disease-resistance. Front. Plant Sci. 2019, 9, 1999. [CrossRef]

39. Yin, H.; Hong, G.; Li, L.; Zhang, X.; Kong, Y.; Sun, Z.; Li, J.; Chen, J.; He, Y. miR156/SPL9 regulates reactive oxygen species accumulation and immune response in Arabidopsis thaliana. Phytopathology 2019, 109, 632-642.

40. Campo, S.; Peris-Peris, C.; Siré, C.; Moreno, A.B.; Donaire, L.; Zytnicki, M.; Notredame, C.; Llave, C.; San Segundo, B. Identification of a novel microRNA (miRNA) from rice that targets an alternatively spliced transcript of the Nramp6 (Natural resistanceassociated macrophage protein 6) gene involved in pathogen resistance. New Phytol. 2013, 199, 212-227. [CrossRef]

41. Li, Y.; Lu, Y.G.; Shi, Y.; Wu, L.; Xu, Y.J.; Huang, F.; Guo, X.Y.; Zhang, Y.; Fan, J.; Zhao, J.Q.; et al. Multiple rice microRNAs are involved in immunity against the blast fungus Magnaporthe oryzae. Plant Physiol. 2014, 164, 1077-1092. [CrossRef] [PubMed]

42. Li, Y.; Zhao, S.L.; Li, J.L.; Hu, X.H.; Wang, H.; Cao, X.L.; Xu, Y.J.; Zhao, Z.X.; Xiao, Z.Y.; Yang, N.; et al. Osa-miR169 negatively regulates rice immunity against the blast fungus Magnaporthe oryzae. Front. Plant Sci. 2017, 8, 2. [CrossRef] [PubMed]

43. Li, Y.; Cao, X.L.; Zhu, Y.; Yang, X.M.; Zhang, K.N.; Xiao, Z.Y.; Wang, H.; Zhao, J.H.; Zhang, L.L.; Li, G.B.; et al. Osa-miR398b boosts $\mathrm{H}_{2} \mathrm{O}_{2}$ production and rice blast disease-resistance via multiple superoxide dismutases. New Phytol. 2019, 222, 1507-1522. [CrossRef] [PubMed]

44. Li, X.P.; Ma, X.C.; Wang, H.; Zhu, Y.; Liu, X.X.; Li, T.T.; Zheng, Y.P.; Zhao, J.Q.; Zhang, J.W.; Huang, Y.Y.; et al. Osa-miR162a fine-tunes rice resistance to Magnaporthe oryzae and yield. Rice 2020, 13, 38. [CrossRef]

45. Salvador-Guirao, R.; Hsing, Y.I.; San Segundo, B. The polycistronic miR166k-166h positively regulates rice immunity via posttranscriptional control of EIN2. Front. Plant Sci. 2018, 9, 337. [CrossRef]

46. Zhang, L.L.; Li, Y.; Zheng, Y.P.; Wang, H.; Yang, X.; Chen, J.F.; Zhou, S.X.; Wang, L.F.; Li, X.P.; Ma, X.C.; et al. Expressing a target mimic of miR156fhl-3p enhances rice blast disease resistance without yield penalty by improving SPL14 expression. Front. Genet. 2020, 11, 327. [CrossRef]

47. Zhang, X.; Bao, Y.; Shan, D.; Wang, Z.; Song, X.; Wang, Z.; Wang, J.; He, L.; Wu, L.; Zhang, Z.; et al. Magnaporthe oryzae Induces the Expression of a MicroRNA to Suppress the Immune Response in Rice. Plant Physiol. 2018, 177, 352-368. [CrossRef]

48. Wang, H.; Li, Y.; Chern, M.; Zhu, Y.; Zhang, L.L.; Lu, J.H.; Li, X.P.; Dang, W.Q.; Ma, X.C.; Yang, Z.R.; et al. Suppression of rice miR168 improves yield, flowering time and immunity. Nat. Plants 2021, 7, 129-136. [CrossRef]

49. Wang, Z.; Xia, Y.; Lin, S.; Wang, Y.; Guo, B.; Song, X.; Ding, S.; Zheng, L.; Feng, R.; Chen, S.; et al. Osa-miR164a targets OsNAC60 and negatively regulates rice immunity against the blast fungus Magnaporthe oryzae. Plant J. 2018, 95, 584-597. [CrossRef]

50. Zhao, Z.X.; Feng, Q.; Cao, X.L.; Zhu, Y.; Wang, H.; Chandran, V.; Fan, J.; Zhao, J.Q.; Pu, M.; Li, Y.; et al. Osa-miR167d facilitates infection of Magnaporthe oryzae in rice. J. Integr. Plant Biol. 2020, 62, 702-715. [CrossRef]

51. Zhou, S.; Zhu, Y.; Wang, L.; Zheng, Y.P.; Chen, J.F.; Li, T.T.; Yang, X.M.; Wang, H.; Li, X.P.; Ma, X.C.; et al. Osa-miR1873 fine-tunes rice immunity against Magnaporthe oryzae and yield traits. J. Integr. Plant Biol. 2019, 62, 1213-1226. [CrossRef]

52. Xiang, Y.; Song, M.; Wei, Z.; Tong, J.; Zhang, L.; Xiao, L.; Ma, Z.; Wang, Y. A jacalin-related lectin-like gene in wheat is a component of the plant defence system. J. Exp. Bot. 2011, 62, 5471-5483. [CrossRef] [PubMed]

53. Cheng, Q.; Dong, L.; Gao, T.; Liu, T.; Li, N.; Wang, L.; Chang, X.; Wu, J.; Xu, P.; Zhang, S. The bHLH transcription factor GmPIB1 facilitates resistance to Phytophthora sojae in Glycine max. J. Exp. Bot. 2018, 69, 2527-2541. [CrossRef] [PubMed]

54. Keith, B.; Dong, X.N.; Ausubel, F.M.; Fink, G.R. Differential induction of 3-deoxy-D-arabino-heptulosonate 7-phosphate synthase genes in Arabidopsis thaliana by wounding and pathogenic attack. Proc. Natl. Acad. Sci. USA 1991, 88, 8821-8825. [CrossRef] [PubMed] 